\title{
Repeatability and reproducibility of horizontal corneal diameter and anterior corneal power measurements using the Oculus Keratograph 4
}

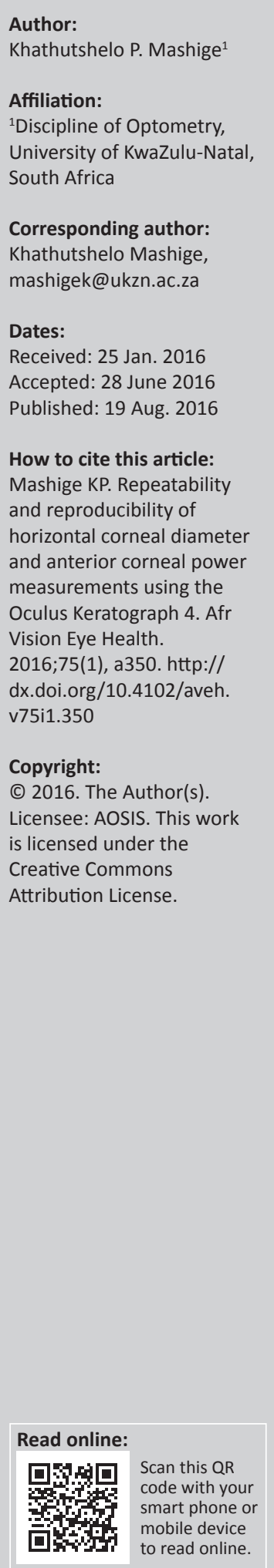

Purpose: To evaluate the repeatability and reproducibility of horizontal corneal diameter (HCD) and anterior corneal power (ACP) measurements obtained with the Oculus Keratograph 4 (OCULUS Optikgeräte $\mathrm{GmbH})$.

Methods: These parameters (HCD and ACP) were prospectively measured in quick succession three times in each of the right eyes of 40 healthy subjects, aged 18-28 years, with normal vision ( $6 / 6$ or better visual acuity) in the first session by a single examiner. Measurements were then repeated in the second session scheduled 1 week later by the same examiner using the same instrument. Repeatability and reproducibility of HCD and ACP measurements was assessed based on the intra-session and intersession within-subject standard deviation $\left(s_{w}\right)$, repeatability $\left(2.77 s_{w}\right)$, coefficient of variation $(\mathrm{CoV})$ and intra-class correlation coefficient (ICC).

Results: Intra-session repeatability and intersession reproducibility of all measured parameters showed a repeatability $\left(2.77 s_{w}\right)$ of $0.35 \mathrm{~mm}$ or less for HCD and $0.35 \mathrm{D}$ or less for ACP, a CoV of $0.30 \%$ or less and an ICC of more than 0.9 .

Conclusion: HCD and ACP measurements obtained using an Oculus Keratograph 4 show good repeatability and reproducibility in healthy eyes; therefore, these parameters can be used for longitudinal follow-up when measured with this device.

\section{Introduction}

It is important for clinicians and researchers to measure corneal diameter with a high degree of precision or accuracy and reliability also to diagnose and manage congenital glaucoma, ${ }^{1,2}$ for intra-ocular lens power calculations, ${ }^{3,4,5}$ and to select and fit contact lenses. ${ }^{6}$ Corneal power measurements also have many important applications in corneal and cataract refractive surgeries, ${ }^{7,8}$ orthokeratology, ${ }^{9}$ ocular aberration analysis as well as for diagnosing and managing keratoconus and contact lens fitting. ${ }^{10}$

The Oculus Keratograph 4 (OCULUS Optikgeräte $\mathrm{GmbH}$ ) uses Placido ring-based videokeratography to provide corneal topography (by reflecting illuminated rings on the cornea). ${ }^{11}$ In addition to corneal topographic measurements, this device has other functions, such as pupillometry, tear assessment, contact lens prediction of posterior surface fit and lid-angle and imaging measurements. ${ }^{11}$ The instrument is commonly used and is an automated and largely examiner-independent technique for corneal topography. ${ }^{11,12}$

Most published studies have focused on comparing different types of corneal topography devices $^{13,14,15}$ and also investigated the repeatability and reproducibility of pachymetric measurements obtained by various instruments, such as the Pentacam, Orbscan and ultrasound. ${ }^{16,17}$ Other studies have focused on comparing these instruments before and after corneal surgery. ${ }^{18,19}$ Studies by Best et al. ${ }^{11}$ and Ortiz-Toquero et al. ${ }^{12}$ found that the Oculus Keratograph 4 provides highly repeatable measurements of corneal topography in healthy eyes; however, the intersession reproducibility of this instrument was not assessed. Another study ${ }^{20}$ found that this device had excellent reliability and high agreement with two other devices for anterior corneal power (ACP) measurements; however, the repeatability and reproducibility of horizontal corneal diameter (HCD) measurements with this device were not assessed. The aim of this study was therefore to determine the intra-session repeatability and intersession reproducibility of the Oculus Keratograph 4 for HCD and ACP measurements in healthy adult eyes. 


\section{Methods}

Forty young adult university students (20 male and 20 female students) with a mean age of $22.6 \pm 3.4$ years (range, 18-28 years), were chosen by convenience sampling for this study. All procedures followed the Declaration of Helsinki, and the protocol was reviewed and approved by the Biomedical Research and Ethics Committee of the University of KwaZulu-Natal. Written informed consent was received from all subjects after the nature of the study had been explained to them. Inclusion criteria were age 18 years or older, a compensated visual acuity of $6 / 6$ or better and a spherical and cylindrical refraction within $\pm 5.0 \mathrm{D}$. Exclusion criteria were any form of ocular pathology (including ocular surface diseases, such as dry eye, conjunctivitis, corneal opacities or dystrophies), any history of ocular surgery, trauma or contact lens wear and use of medication that could affect corneal ocular physiology, all of which can result in abnormal measurements. Each subject underwent a full ophthalmic examination including vision, auto-refraction and subjective-refraction, slit-lamp examination, non-contact tonometry, fundus examination and corneal topography measurements with the Oculus Keratograph 4.

For the purposes of this study, the following corneal indices from the Oculus Keratograph 4 assessment were used: HCD (horizontal corneal, limbus-to-limbus, diameter), $\mathrm{Kf}$ (simulated keratometry in the flattest meridian in the 3.00-mm zone) and Ks (simulated keratometry in the steepest meridian in the $3.00-\mathrm{mm}$ zone). ${ }^{12}$ The vector presentation included $\mathrm{M}$ (mean power of the flattest and steepest corneal meridians), $\mathrm{J}_{0}$ (corneal astigmatism along $90^{\circ} / 180^{\circ}$ ) and $\mathrm{J}_{45}$ (corneal astigmatism along $45^{\circ} / 135^{\circ}$ ). M also represents the spherical equivalent of a refraction. ${ }^{21}$ The Oculus Keratograph 4 calculates both $\mathrm{Kf}$ and $\mathrm{Ks}$ values by entering the value of anterior corneal curvature radius (R) according to the formula $\mathrm{D}=(1.3375-1) \times(1000) / \mathrm{R} \mathrm{mm} .{ }^{12}$ Calibration of the device was performed by the manufacturer prior to data collection.

Corneal topography data were taken between $4 \mathrm{pm}$ and $8 \mathrm{pm}$ in both sessions, with all subjects having been awake for at least 3 hours beforehand. The eye is most physiologically stable between $4 \mathrm{pm}$ and $8 \mathrm{pm}^{22,23}$; therefore, this time was chosen to ensure that corneal diurnal and nocturnal changes did not influence the measurements. The subjects were also requested to avoid substantial reading prior to the measurements. ${ }^{24}$ Only the right eye of each subject was selected. During the first of the two sessions, three sets of measurements per eye were performed by a single experienced examiner for all subjects according to the manufacturers' instructions (intra-session repeatability). The time interval between each measurement was kept as short as possible. Subjects were instructed to blink completely just before each scan to spread an optically smooth tear film over the cornea as it has been reported that Placido-based corneal topographers are affected by tear-film instability. ${ }^{20}$ To eliminate interdependence of the successive measurements, they were requested to move their chin from the chinrest between scans. ${ }^{12}$ They were asked to sit back after each repeat scan, with the device being realigned before each one. Measurements (i.e. three per eye) were repeated in the second session that was scheduled 1 week later at a similar time as the first session and was conducted by the same examiner using the same protocol (intersession reproducibility).

\section{Statistical analysis}

Statistical analysis was performed using SPSS software for Windows version 19 (SPSS Inc., Chicago, IL, USA). In this study, repeatability and reproducibility of HCD and ACP were calculated based on the definitions adopted by the British Standards Institute and the International Organisation for Standardisation, ${ }^{25,26}$ as recommended by Bland and Altman. ${ }^{27}$ The distributions of the data sets were checked for normality using Kolmogorov-Smirnov tests. The results indicated that the data were normally distributed $(p>0.05)$. For each scan, HCD, the flat (Kf), steep (Ks) and mean (M) corneal values and the axes of $\mathrm{Kf}$ and $\mathrm{Ks}$ were acquired. Corneal astigmatism was converted into a vector representation of Jackson $\mathrm{J}_{0}$ and $\mathrm{J}_{45^{\prime}}$ by using the following formula as recommended by Thibos et al. ${ }^{28}$ :

$\mathrm{J}_{0}=($-cylinder $/ 2) \cos (2 \times$ axis $)$

[Eqn 1]

$\mathrm{J}_{45}=($-cylinder $/ 2 \sin (2 \times$ axis $)$

'In order to investigate the repeatability of measurements, a repeatability study must, for an appropriately selected sample, make at least two measurements per subject under identical conditions'. ${ }^{29}$ To assess the repeatability of three repeated measurements on the 40 subjects, a one-way analysis of variance (ANOVA) was performed to determine the within-subject standard deviation $\left(s_{w}\right)$. Test-retest repeatability, within-subject coefficient of variation $(\mathrm{CoV})$ and intra-class correlation coefficients (ICCs) were also calculated for the three measurements obtained in each of the two sessions to determine the applicable intrasession repeatability. Test-retest repeatability (defined as $1.96 \sqrt{2} \times s_{w}=2.77 s_{w}$ ) is an interval within which $95 \%$ of the differences between measurements are expected to lie. ${ }^{30}$ The $\mathrm{CoV}$ was calculated using the $s_{w}$ divided by the overall mean, expressed as a percentage $\left[\mathrm{CoV}=s_{w} /\right.$ mean $\left.\times 100(\%)\right] .{ }^{30} \mathrm{ICC}$ was calculated based on the repeated-measures ANOVA..$^{30}$ The differences between the three measurements were determined with one-way ANOVA and $p$-values less than 0.05 were considered statistically significant.

Similarly, to assess intersession reproducibility, the mean of the three readings per eye from each session was firstly calculated for each parameter, after which the intersession $s_{w}, 2.77 s_{w}, \mathrm{CoV}$ and ICCs were calculated. The advantage of $\mathrm{CoV}$ values is that they can be compared between data sets with different units or widely different means. ${ }^{31,32}$ The disadvantage is that when the mean value is near zero, the $\mathrm{CoV}$ is sensitive to small changes in the mean, limiting its usefulness. ${ }^{31,32}$ In this study, the mean values of $\mathrm{J}_{0}$ and $\mathrm{J}_{45}$ were both near zero; therefore, their CoVs were not calculated. 


\section{Results}

The mean spherical equivalent refractive error of the enrolled participants was $-0.50 \mathrm{D} \pm 2.16 \mathrm{D}$ (range, $0.50 \mathrm{D}$ to $-4.75 \mathrm{D}$ ). The repeatability during the first and second sessions of all parameters assessed was high (see Table 1), with the $2.77 s_{w}$ of repeated $\mathrm{HCD}, \mathrm{Kf}, \mathrm{Ks}$ and $\mathrm{M}$ measurements less than 0.36 . The CoV were smaller than $0.31 \%$ and ICCs were higher than 0.9. The M had the highest ICC and the lowest CoV (see Table 1). There were no statistically significant differences between the three measurements per session in both HCD and ACP ( $p>0.05$, ANOVA), suggesting that there is no difference between the means of the measurements across the 40 eyes.

There were no significant differences in the measurements between the first and the second sessions (Table 2). The CoV values of HCD were $0.25 \%$, and the $s_{w}$ and $2.77 s_{w}$ values were $0.07 \mathrm{~mm}$ and $0.13 \mathrm{~mm}$, respectively. The $\mathrm{CoV}$ values of ACP were less than $0.30 \%$, and the $s_{w}$ and $2.77 s_{w}$ values were within $0.13 \mathrm{D}$ and $0.36 \mathrm{D}$, respectively. The $s_{w}$ and $2.77 s_{w}$ of $\mathrm{J}_{0}$ and $\mathrm{J}_{45}$ values were within $0.15 \mathrm{D}$ and $0.29 \mathrm{D}$, respectively, and the ICCs of all parameters were above 0.924 .

The CoV values of the HCD and ACP were less than $0.30 \%$, and the ICC values were more than 0.9 for all parameters assessed. The ICC ranges from 0 to 1 and measures the consistency for data sets of repeated measurements according to the classification proposed by Fermanian. ${ }^{32}$ Concordance is excellent for ICC $>0.91$, good for ICC ranging between 0.90 and 0.71 , moderate for ICC ranging between 0.70 and 0.51 , fair for ICC ranging between 0.50 and 0.31 and bad for ICC $<0.30 .^{33}$ Excellent repeatability was found in the HCD and ACP and there was no statistically significant differences between these measurements $(p>0.05$, ANOVA) in all topographical outcomes. Therefore, the Oculus Keratograph 4 showed high intra-session repeatability in measuring $\mathrm{HCD}$ and ACP.

\section{Discussion}

Accurate and precise determination of HCD and ACP are fundamental and important to many clinical and research applications. ${ }^{3,4,5,6,7,8,9,10}$ Repeatability and reproducibility are two important components of precision in any measurement system. ${ }^{25,26,27}$ Repeatability refers to the variability of at least two measurements taken by a single person on the same subjects, measuring the same item repeatedly, using the same instrument under similar conditions. ${ }^{27}$ Reproducibility is the variability of the measurements obtained on the same subject during different sessions under the same conditions. ${ }^{25,26,27}$ In this study, the intra-session repeatability and intersession reproducibility of $\mathrm{HCD}$ and $\mathrm{ACP}$ measurements were assessed with the Oculus Keratograph 4. The results of this study showed high intra-session repeatability and high intersession reproducibility of the Oculus Keratograph 4 measurement for these parameters.

\section{Intra-session repeatability}

The HCD repeatability results obtained in this study confirm the data reported by Ortiz-Toquero et al. ${ }^{12}$ The ACP repeatability results are similar to those reported using other Placido disk-based corneal topographers. ${ }^{34,35}$ For example,

TABLE 1: Intra-session reproducibility of horizontal corneal diameter, flattest and steepest keratometry and power vectors $\mathrm{M}$, $\mathrm{J}_{0}$ and $\mathrm{J}_{45}$ using the Oculus Keratograph 4.

\begin{tabular}{|c|c|c|c|c|c|c|c|c|c|}
\hline \multirow[t]{2}{*}{ Parameter } & \multirow[t]{2}{*}{ Session } & \multicolumn{2}{|c|}{ Mean \pm s.d. } & \multicolumn{2}{|c|}{$s_{w}$} & \multicolumn{2}{|c|}{$2.77 s_{w}$} & \multirow[t]{2}{*}{$\operatorname{CoV}(\%)$} & \multirow[t]{2}{*}{ ICC } \\
\hline & & $\mathrm{mm}$ & D & $\mathrm{mm}$ & D & $\mathrm{mm}$ & D & & \\
\hline \multirow[t]{2}{*}{$\mathrm{HCD}$} & 1 & $11.78 \pm 0.47$ & - & 0.07 & - & 0.16 & - & 0.26 & 0.986 \\
\hline & 2 & $11.81 \pm 0.45$ & - & 0.09 & - & 0.14 & - & 0.28 & 0.989 \\
\hline \multirow[t]{2}{*}{$\mathrm{Kf}$} & 1 & - & $42.66 \pm 1.21$ & - & 0.14 & - & 0.33 & 0.30 & 0.994 \\
\hline & 2 & - & $42.69 \pm 1.23$ & - & 0.11 & - & 0.28 & 0.26 & 0.987 \\
\hline \multirow[t]{2}{*}{ Ks } & 1 & - & $43.51 \pm 1.36$ & - & 0.12 & - & 0.34 & 0.28 & 0.992 \\
\hline & 2 & - & $43.52 \pm 1.35$ & - & 0.13 & - & 0.35 & 0.29 & 0.992 \\
\hline \multirow[t]{2}{*}{ M } & 1 & - & $43.07 \pm 1.25$ & - & 0.11 & - & 0.32 & 0.24 & 0.993 \\
\hline & 2 & - & $43.09 \pm 1.27$ & - & 0.10 & - & 0.29 & 0.22 & 0.994 \\
\hline \multirow[t]{2}{*}{$\mathrm{J}_{0}$} & 1 & - & $-0.33 \pm 0.22$ & - & 0.09 & - & 0.19 & - & 0.969 \\
\hline & 2 & - & $-0.33 \pm 0.21$ & - & 0.08 & - & 0.17 & - & 0.978 \\
\hline \multirow[t]{2}{*}{$\mathrm{J}_{45}$} & 1 & - & $0.00 \pm 0.11$ & - & 0.07 & - & 0.15 & - & 0.918 \\
\hline & 2 & - & $0.00 \pm 0.11$ & - & 0.06 & - & 0.12 & - & 0.934 \\
\hline
\end{tabular}

HCD, horizontal corneal diameter; Kf, flattest; Ks, steepest; D, dioptre; SD, standard deviation; $s_{w}$, within-subject standard deviation; CoV, within-subject coefficient of variation; ICC, intra-class correlation coefficient.

TABLE 2: Intersession reproducibility of horizontal corneal diameter, flattest and steepest keratometry and power vectors $\mathrm{M}$, $\mathrm{J}_{0}$ and $\mathrm{J}_{45}$ using the Oculus Keratograph 4.

\begin{tabular}{|c|c|c|c|c|c|c|c|c|}
\hline \multirow[t]{2}{*}{ Parameter } & \multicolumn{2}{|c|}{ Mean \pm s.d. } & \multicolumn{2}{|c|}{$s_{w}$} & \multicolumn{2}{|c|}{$2.77 s_{w}$} & \multirow[t]{2}{*}{$\operatorname{CoV}(\%)$} & \multirow[t]{2}{*}{ ICC } \\
\hline & $\mathrm{mm}$ & D & $\mathrm{mm}$ & D & $\mathrm{mm}$ & D & & \\
\hline $\mathrm{HCD}$ & $11.80 \pm 0.49$ & - & 0.07 & - & 0.13 & - & 0.25 & 0.991 \\
\hline $\mathrm{Kf}$ & - & $42.64 \pm 1.23$ & - & 0.10 & - & 0.27 & 0.25 & 0.986 \\
\hline Ks & - & $43.51 \pm 1.38$ & - & 0.12 & - & 0.35 & 0.29 & 0.990 \\
\hline M & - & $43.09 \pm 1.25$ & - & 0.08 & - & 0.30 & 0.24 & 0.993 \\
\hline $\mathrm{J}_{0}$ & - & $-0.31 \pm 0.23$ & - & 0.11 & - & 0.28 & - & 0.974 \\
\hline $\mathrm{J}_{45}$ & - & $0.00 \pm 0.13$ & - & 0.14 & - & 0.33 & - & 0.925 \\
\hline
\end{tabular}

HCD, horizontal corneal diameter; Kf, flattest; Ks, steepest; D, dioptre; SD, standard deviation; $s_{w^{\prime}}$ within-subject standard deviation; CoV, within-subject coefficient of variation; ICC, intra-class correlation coefficient. 
Wang et al. ${ }^{34}$ found excellent repeatability of the Topolyzer (Wavelight Technologie AG), with a $2.77 s_{w}$ of less than $0.35 \mathrm{D}$, less than $0.36 \mathrm{D}$ and ICC of more than 0.99 , for Kf, Ks, and M, respectively. The Oculus Keratograph 4 and Topolyzer have higher resolutions because they have 22 rings and measure 22000 data points compared to other Placido-based devices, such as the Eyesys Vista instrument which has 26 rings but only measures 9360 points. ${ }^{20}$ Mao et al. ${ }^{20}$ suggested that it is likely that more rings and data points improve the reliability of measurements. This study included subjects with relatively small magnitudes of astigmatism, which could have influenced this result. Chen and $\mathrm{Lam}^{32}$ also found that the repeatability of vector components $\mathrm{J}_{0}$ and $\mathrm{J}_{45}$ is slightly variable.

\section{Intersession reproducibility}

As in the intra-session repeatability, the $s_{w}$ and $2.77 s_{w}$ values of $\mathrm{HCD}, \mathrm{Kf}, \mathrm{Ks}$, and $\mathrm{M}$ were within acceptable limits (see Table 2), suggesting that there were no significant differences in the measurements between the first and second sessions. This study has shown for the first time that the Oculus Keratograph 4 provides highly reproducible measurements of HCD, as evidenced by the low $S_{w w}(<0.15 \mathrm{~mm})$ and high ICC (>0.92) values. In addition, this device offers excellent reproducibility for ACP measurements.

Huang et $a l .^{35}$ found similar results with the OphthaTOP Placido disk-type corneal topographer, where the maximum $2.77 s_{w}$, maximum $\mathrm{CoV}$, and minimum ICC values were $0.24 \mathrm{D}$, $0.20 \%, 0.942 \mathrm{D}$ and $0.29 \mathrm{D}, 0.24 \%, 0.921$, respectively, for ACP measurements. In addition, the results of the present study are comparable to those obtained by the Topolyzer. ${ }^{34}$ The Topolyzer displayed excellent reproducibility in measuring ACP (ICCs $\geq 0.971$ ) and astigmatism (ICCs $>0.97$ for both $\mathrm{J}_{0}$ and $\mathrm{J}_{45}$ ). Because of its high precision, the Topolyzer has been reported to be an effective and safe tool in topographyguided corneal excimer laser surgery to correct refractive errors. $^{36}$ The CoV and ICC values were less than $0.30 \%$ and higher than 0.91, respectively, indicating excellent reproducibility of the Oculus Keratograph 4 for HCD and ACP measurements. The Oculus Keratograph 4 also offered excellent, although slightly lower, reproducibility for astigmatic vector analysis. These results are similar to those obtained by other Placido-based corneal topographers, such as the OphthaTOP ${ }^{36}$ and Keratron (Optikon 2000 SpA, Rome, Italy). ${ }^{37}$

The present study was conducted on a young adult population with good vision, which ensured a good image capture with the Oculus Keratograph 4. Further studies are needed to evaluate the repeatability and reproducibility of this device in older subjects and children, as well as in those with corneal and/or other ocular pathologies. In summary, the Oculus Keratograph 4 provides non-invasive, repeatable and reproducible measurements of HCD and ACP in healthy eyes. Its automatic measurement activation guarantees fast and accurate measurements and can significantly streamline the workflow in a clinical setting.

\section{Acknowledgements}

This work is part of the $\mathrm{PhD}$ vision science project funded by the Medical Research Council, South Africa. The author thanks Professor O.A. Oduntan and Ms Carrin Martin for their help throughout this project.

\section{Competing interests}

The author declares that he has no financial or personal relationships which may have inappropriately influenced him in writing this article.

\section{References}

1. Wallace DK, Plager DA. Corneal diameter in childhood aphakic glaucoma. J Pediatr Ophthalmol Strabismus. 1996;33:230-234.

2. Rufer F, Schroder A, Erb C. White-to-white corneal diameter: Normal values in healthy humans obtained with the Orbscan II topography system. Cornea. 2005;24:259-261. http://dx.doi.org/10.1097/01.ico.0000148312.01805.53

3. Ashaye AO, Olowu JA, Adeoti CO. Corneal diameters in infants born in two hospitals in Ibadan, Nigeria. East Afr Med J. 2006;83:631-636.

4. Durukan AH, Mutlu FM, Sahin OF, Altinsoy HI, Bayer A, Celik Y. The importance of corneal diameter in cases developing glaucoma after childhood cataract surgery. Gulhane Med J. 2005;47:94-96.

5. Lee AC, Qazi MA, Pepose JS. Biometry and intraocular lens power calculation. Curr Opin Ophthalmol. 2008;19:13-17. http://dx.doi.org/10.1097/ICU.0b013e3282 f1c5ad

6. Lloyd McKerman A, O'Dwyer V, Simo Manion L. The influence of soft contact lens wear and two weeks cessation of lens wear on corneal curvature. Cont Lens Anterior Eye. 2014;37:31-37. http://dx.doi.org/10.1016/j.clae.2013.07.014

7. Parede TR, Torricelli AA, Mukai A, Vieira Netto M, Bechara SJ. Quality of vision in refractive and cataract surgery, indirect measurers: Review article. Arq Bras Oftalmol. 2013;76:386-390. http://dx.doi.org/10.1590/S0004-274920130006 00016

8. Frings A, Katz T, Steinberg J, Druchkiv V, Richard G, Linke SJ. Ocular residual astigmatism: Effects of demographic and ocular parameters in myopic laser in situ keratomileusis. J Cataract Refract Surg. 2014;40:232-238. http://dx.doi. org/10.1016/j.jcrs.2013.11.015

9. Chui WS, Cho P. A comparative study of the performance of different corneal topographers on children with respect to orthokeratology practice. Optom Vis Sci. 2005;82:420-427. http://dx.doi.org/10.1097/01.0PX.0000162642.24885.71

10. Kamiya K, Ishii R, Shimizu K, Igarashi A. Evaluation of corneal elevation, pachymetry and keratometry in keratoconic eyes with respect to the stage of Amsler-Krumeich classification. Br J Ophthalmol. 2014;98:459-463. http://dx.doi.org/10.1136/ bjophthalmol-2013-304132

11. Best N, Drury L, Wolffsohn JS. Clinical evaluation of the Oculus Keratograph. Cont Lens Anterior Eye. 2012;35:171-174. http://dx.doi.org/10.1016/j.clae.2012.04.002

12. Ortiz-Toquero S, Rodriquez G, de Juan V, Martin R. Repeatability of Placido-based corneal topography in Keratoconus. Optom Vis Sci. 2014;91:1467-1473. http:// dx.doi.org/10.1097/OPX.0000000000000421

13. Baumeister M, Terzi E, Ekiei Y, Ekiei Y. Comparison of manual and automated methods to determine horizontal corneal diameter. J Cataract Refract Surg. 2004;30:374-380. http://dx.doi.org/10.1016/j.jcrs.2003.06.004

14. Salouti R, Nowroozzadeh MH, Zamani M, Ghoreyshi M, Salouti R. Comparison of horizontal corneal diameter measurements using Galilei, EyeSys and Orbscan II systems. Clin Exp Optom. 2009:92:429-433. http://dx.doi.org/10.1111/j.14445ystems. Clin Exp Opt

15. Martin R, Ortiz S, Rio-Cristobal A. White-to-white corneal diameter differences in moderately and highly myopic eyes: Partial coherence inferometry versus scanning-slit topography. J Cataract Refract Surg. 2013;39:585-589. http://dx.doi. org/10.1016/j.jcrs.2012.11.021

16. Fam HR, Lim KL, Reinstein DZ. Orbscan global pachymetry: Analysis of repeated measures. Optom Vis Sci. 2005;82:1047-1053. http://dx.doi.org/10.1097/01. opx.0000192348.37026.09

17. Jonuscheit S, Doughty MJ. Regional repeatability measures of corneal thickness: Orbscan II and Ultrasound. Optom Vis Sci. 2007;84:52-58. http://dx.doi. org/10.1097/01.opx.0000254045.62252.b4

18. Matsuda J, Hieda O, Kinoshita S. Comparison of central corneal thickness measurements by Orbscan II and Pentacam after corneal refractive surgery. Jpn Ophthalmol. 2008;52:245-249. http://dx.doi.org/10.1007/s10384-008-0550-x

19. Hashemi H, Mehravaran S. Central corneal thickness measurements with Pentacam, Orbscan II and ultrasound devices before and after laser refractive surgery for myopia. J Cataract Refract Surg. 2007;33:1701-1707. http://dx.doi org/10.1016/j.jcrs.2007.05.040

20. Mao X, Savini G, Zhuo Z, et al. Repeatability, reproducibility, and agreement of corneal power measurements obtained with a new corneal topographer. Cataract Refract Surg. 2013;39:1561-1569. http://dx.doi.org/10.1016/j.jcrs.2013. 04.029 
21. Harris WF. Algebra of sphere-cylinder and refractive errors, and their means, variance, and standard deviations. Am J Optom Physiol Opt. 1988;65:794-802. $\mathrm{http}: / / \mathrm{dx}$.doi.org/10.1097/00006324-198810000-00003

22. Aakre BM, Doughty MJ, Dalane OV, Berg A, Aamodt O, Gangstad H. Assessment of reproducibility of measures of intraocular pressure and central corneal thickness in young white adults over a 16-h time period. Ophthal Physiol Opt. 2003;23:271-283. http://dx.doi.org/10.1046/j.1475-1313.2003.00117.x

23. Doughty MJ, Zaman ML. Human corneal thickness and its impact on intraocular pressure measures: A review and metaanalysis approach. Surv Ophthalmol. 2000;44:367-408. http://dx.doi.org/10.1016/S0039-6257(00)00110-7

24. Collins MJ, Buehren T, Bece A, Voetz SC. Corneal optics after reading, microscopy and computer work. Acta Ophthalmol Scand. 2006;84:216-224. http://dx.doi. org $/ 10.1111 / \mathrm{j} .1600-0420.2005 .00547 x$

25. British Standards Institution. Accuracy (Trueness and Precision) of measurement methods and results: General principles and definitions. London: HMO BS ISO 5725 part 1; 1994.

26. British Standards Institution. Accuracy (Trueness and Precision) of measurement methods and results: Basic methods for the determination of repeatability and reproducibility of a standard measurement method. London: HMO BS ISO 5725 part 2; 1994.

27. Bland JM, Altman DG. Statistical methods for assessing agreement between two methods of clinical measurement. Lancet. 1986;8:307-310. http://dx.doi. org/10.1016/S0140-6736(86)90837-8

28. Thibos LN, Wheeler W, Horner D. Power vectors: An application of Fourier analysis to the description and statistical analysis of refractive error. Optom Vis Sci. 1997;74:367-375. http://dx.doi.org/10.1097/00006324-199706000-00019
29. Bartlett JW, Frost C. Reliability, repeatability and reproducibility: Analysis of measurement errors in continuous variables. Ultrasound Obstet Gynecol. 2008;31:466-475. http://dx.doi.org/10.1002/uog.5256

30. Bland JM, Altman DG. Measuring agreement in method comparison studies. Stat Methods Med Res. 1999;8:135-160. http://dx.doi.org/10.1191/09622809967381 9272

31. Wang L, Shirayama M, Koch DD. Repeatability of corneal power and wavefront aberration measurements with a dual-Scheimpflug Placido corneal topographer. Cataract Refract Surg. 2010;36:425-430. http://dx.doi.org/10.1016/j.jcrs.2009.09.034

32. Chen D, Lam AKC. Reliability and repeatability of the Pentacam on corneal curvatures. Clin Exp Optom. 2009;92:110-118. http://dx.doi.org/10.1111/j.14440938.2008.00336.x

33. Fermanian J. Mesure de l'accordentre deux juges: Cas quantitatif. Rev Epidém Santé Publ. 1984;32:408-413.

34. Wang Q, Savini G, Hoffer KJ, et al. A comprehensive assessment of the precision and agreement of anterior corneal power measurements obtained using 8 different devices. PLoS One. 2012;7:e45607. http://dx.doi.org/10.1371/journal.pone.0045607

35. Huang J, Savini G, Chen $\mathrm{H}$, et al. Precision and agreement of corneal power measurements obtained using a new corneal topographer OphthaTOP. PLoS One. 2015;10(1):e109414. http://dx.doi.org/10.1371/journal.pone.0109414

36. Falavarjani KG, Hashemi M, Modarres M, Sanjari MS, Darvish N, Gordiz A Topography-guided vs wavefront-optimized surface ablation for myopia using the http://dx.doi.org/10.3928/1081597X-20100310-02

37. Kawamorita T, Nakayama N, Uozato H. Repeatability and reproducibility of corneal curvature measurements using the Pentacam and Keratron topography systems. J Refract Surg. 2009;25:539-544. 